\title{
PADRÕES DE AUTOCORRELAÇÃO ESPACIAL DE ÍNDICES DE VEGETAÇÃO MODIS NO BIOMA CERRADO'
}

\author{
Fábio Martins Vilar de Carvalho ${ }^{2,4}$, Laerte G. Ferreira ${ }^{3}$, Fabio C. Lobo ${ }^{3}$, José Alexandre Felizola Diniz- \\ Filho $^{4}$ e Luís Maurício Bini ${ }^{4}$
}

\begin{abstract}
RESUMO - Embora os índices de vegetação MODIS estejam sendo extensivamente investigados quanto ao seu potencial para o mapeamento e monitoramento biofísico do bioma Cerrado, em particular no que diz respeito à sazonalidade e fenologia da cobertura vegetal, pouco se sabe sobre o comportamento espacial desses índices em escalas regionais. Assim, neste estudo foram avaliados, à escala adotada em estudos de macroecologia (Resolução de $1^{\circ} \times 1^{\circ}$ ), os padrões de autocorrelação espacial do EVI (índice de vegetação realçado) e NDVI (índice de vegetação da diferença normalizada), utilizando-se índices $I$ de Moran obtidos em diferentes classes de distância geográfica (correlogramas espaciais). Em média, os valores apresentados por esses índices são autocorrelacionados até uma distância aproximada de $800 \mathrm{~km}$, que pode revelar um padrão de manchas afetado por variação ambiental e conversão da vegetação nativa. No entanto, esses padrões de similaridade espacial são principalmente influenciados pelo contraste sazonal encontrado no bioma Cerrado, bem como em função dos padrões de cobertura da terra e do tipo de índice considerado (i.e., EVI ou NDVI).
\end{abstract}

Palavras-chave: Índices de vegetação MODIS, autocorrelação espacial e Cerrado.

\section{SPATIAL AUTOCORRELATION PATTERNS OF THE MODIS VEGETATION INDICES FOR THE CERRADO BIOME}

\begin{abstract}
While the MODIS vegetation indices have been extensively investigated regarding their potential for mapping and biophysical monitoring of the Cerrado biome, particularly with respect to the seasonality and phenology of the vegetative cover, very little is known about the spatial behavior of these indices. Thus, this study assessed, at the "macroecology" scale ( $1^{\circ} \times 1^{\circ}$ spatial resolution), the autocorrelation patterns of both the EVI (enhanced vegetation index) and NDVI (normalized difference vegetation index), using Moran's I coefficients obtained for several geographic distance classes (spatial correlogram). On average, the values presented by these two indices are correlated up to a distance of about $800 \mathrm{~km}$, possibly revealing a patch pattern affected by environmental variables and native vegetation conversion. On the other hand, these spatial similarity patterns are mainly influenced by the marked seasonal contrast of the cerrado biome, as well as by the land cover classes and the vegetation index considered (i.e. EVI or NDVI).
\end{abstract}

Keywords: MODIS vegetation indices, spatial autocorrelation and Cerrado.

\footnotetext{
${ }^{1}$ Recebido em 21.12.2006 e aceito para publicação em 20.02.2008.

${ }^{2}$ Programa de Pós-Graduação em Ciências Ambientais da Universidade Federal de Goiás (UFG). E-mail: <fmvcarvalho@ yahoo.com.br>.

${ }^{3}$ Laboratório de Processamento de Imagens e Geoprocessamento, Instituto de Estudos Sócio-Ambientais da UFG E-mail: <laerte@iesa.ufg.br>.

${ }^{4}$ Laboratório de Ecologia Teórica e Síntese,Instituto de Ciências Biológicas da UFG. E-mail: <diniz@ @icb.ufg.br > e < bini@icb.ufg.br>.
} 


\section{INTRODUÇÃO}

O sensoriamento remoto, como fonte regular de obtenção de dados da superfície terrestre, constituise numa importante técnica para o monitoramento sistemático da dinâmica da vegetação. Em particular, os índices de vegetação, resultado de combinações lineares de dados espectrais, realçam o sinal da vegetação, ao mesmo tempo que minimizam as variações na irradiância solar e os efeitos do substrato do dossel vegetal (JACKSON e HUETE, 1991). Nesse sentido, possibilitam comparações espaciais e temporais da atividade fotossintética terrestre, facilitando, assim, o monitoramento sazonal, interanual e variações de longo prazo dos parâmetros estruturais, fenológicos e biofísicos da vegetação (WANG et al., 2003).

Um dos índices de vegetação mais utilizados tem sido o Índice de Vegetação da Diferença Normalizada (NDVI), cuja simplicidade e alta sensibilidade à densidade da cobertura vegetal tornaram possível o monitoramento da vegetação em escala global, resultando, até o momento, em mais de 22 anos de dados NDVI obtidos das séries NOAA-AVHRR (HUETE et al., 2002). Embora extremamente útil na estimativa de parâmetros biofísicos da vegetação, o NDVI apresenta problemas de saturação em áreas densamente vegetadas e não-linearidade nas relações com o índice de área foliar (LAI) e com a fração de radiação fotossinteticamente ativa absorvida (fAPAR). O NDVI é também seriamente afetado pela geometria de aquisição (sol-alvo-sensor), a estrutura do dossel vegetal, efeitos atmosféricos e substrato (ex. solo) (GOWARD et al., 1991; MOODY e STRAHLER, 1994). Nesse sentido, Huete et al. (1997) propuseram o Índice de Vegetação Realçado (EVI), que, comparativamente ao NDVI, é menos sensível às contaminações do substrato e da atmosfera (LIU e HUETE, 1995; MIURA et al., 1998, 2001), respondendo melhor às variações fisionômicas e estruturais no dossel (GAO et al., 2000).

No âmbito do sensor MODIS, "carro-chefe" do programa Sistema de Observação Terrestre (EOS - NASA) (JUSTICE et al., 1998), os produtos MOD13 (índices de vegetação), produzidos a cada 16 dias com resoluções de 250,500 e $1.000 \mathrm{~m}$, incluem tanto uma continuidade do NDVI quanto o EVI (HUETE et al., 2002). A disponibilidade dessas informações pode ser importante para a análise dos padrões espaciais de distribuição da vegetação, com conseqüiências para a compreensão de padrões de distribuição da biodiversidade em sistemas ameaçados, como o Cerrado brasileiro. Nesse bioma, diferentes estudos já têm demonstrado a capacidade dos índices de vegetação MODIS para o mapeamento das fisionomias existentes (FERREIRA et al., 2004), bem como em responder aos parâmetros biofísicos (FERREIRA et al., 2003; RATANA et al., 2005) e às variações sazonais - fenológicas da cobertura vegetal (FERREIRA e HUETE, 2004; FERREIRA et al., 2006a).

Da mesma forma, os índices de vegetação são também úteis em estudos de biologia da conservação e macroecologia. Esses podem ser usados, por exemplo, para monitorar desmatamentos e mudanças no uso da terra (FERREIRA et al., 2006b; GOMES et al., 2006) ou para identificar habitats adequados para a preservação de espécies (KERR e OSTROVSKY, 2003; WESSELS et al., 2004; KOY et al., 2005). Em estudos macroecológicos, o NDVI é comumente usado como indicador da produção primária líquida ou heterogeneidade espacial da vegetação, sendo empregado como uma variável ambiental que possibilita explicar o gradiente de riqueza de espécies em grandes escalas (HURLBERT e HASKELL, 2003; RUGGIERO e KITZBERGER, 2004; TOGNELLI e KELT, 2004; HAWKINS et al., 2005). Todos esses estudos estão limitados pelo baixo nível de resolução nas informações sobre distribuição da biodiversidade e, por isso, são restritos a análises na escala de quadrículas com $1^{\circ}$ de aresta, que representa uma escala-padrão para os estudos de macroecologia (DINIZ-FILHO e SANT'ANA, 1998; RODRIGUEZ et al., 2006; DINIZ-FILHO et al., 2007; HAWKINS et al., 2007).

Um fator importante a ser considerado em estudos dessa natureza é a presença de autocorrelação espacial. A maioria dos fenômenos ecológicos, bem como as variáveis físicas que descrevem o ambiente, está sujeita à autocorrelação espacial, e esta pode estar presente qualquer que seja a escala considerada (LEGENDRE e FORTIN, 1989; LEGENDRE, 1993; LENNON, 2000; LACERDA e KAGEYAMA, 2003). A autocorrelação espacial pode ser definida como a existência de uma correlação positiva (autocorrelação positiva) ou negativa (autocorrelação negativa) entre a distância entre pontos e a similaridade nos valores medidos em uma variável em uma intensidade maior que a esperada pelo acaso (LEGENDRE, 1993). Portanto, uma variável é autocorrelacionada se uma medida feita num ponto carrega informação sobre outra medida daquela variável registrada em outro ponto, localizado a determinada 
distância. Dessa forma, os valores não são independentes do ponto de vista estatístico, violando um pressuposto importante dos testes tradicionais, i.e., o da independência dos dados entre os pontos de amostragem (CALEGARIO et al., 2005). Assim, os padrões de autocorrelação espacial nos dados podem criar resultados falso-positivos nas análises (DINIZ-FILHO et al., 2003). A presença de autocorrelação espacial nos dados indica que parte da variância da variável estudada é explicada pelo efeito da variabilidade espacial (LEGENDRE, 1993; SELMI e BOULINIER, 2001).

O objetivo principal deste estudo foi descrever a estrutura espacial dos índices de vegetação MODIS NDVI e EVI no bioma Cerrado, na escala espacial usualmente adotada em estudos de macroecologia (com quadrículas de $1^{\circ}$ de aresta). Foram avaliados o grau de autocorrelação espacial e, mais especificamente, a dependência dos padrões espaciais observados em relação ao tipo de índice utilizado, à sazonalidade da cobertura vegetal e às classes de cobertura da terra consideradas.

\section{MATERIAL E MÉTODOS}

\subsection{Dados}

Neste estudo foram empregadas cinco cenas (tiles) do produto MOD13Q1 (h12/13 v09/11), correspondentes ao limite do Cerrado (IBGE, 2004) (Figura 1), no ano de 2005, obtidas na primeira quinzena do mês de maio (período úmido) e do mês de setembro (período seco). Após a realização de mosaicos referentes à área de estudo, os conjuntos de imagens foram filtrados (para nuvens, sombras e aerossóis residuais), com base no respectivo Quality Assurance layer (metadados).

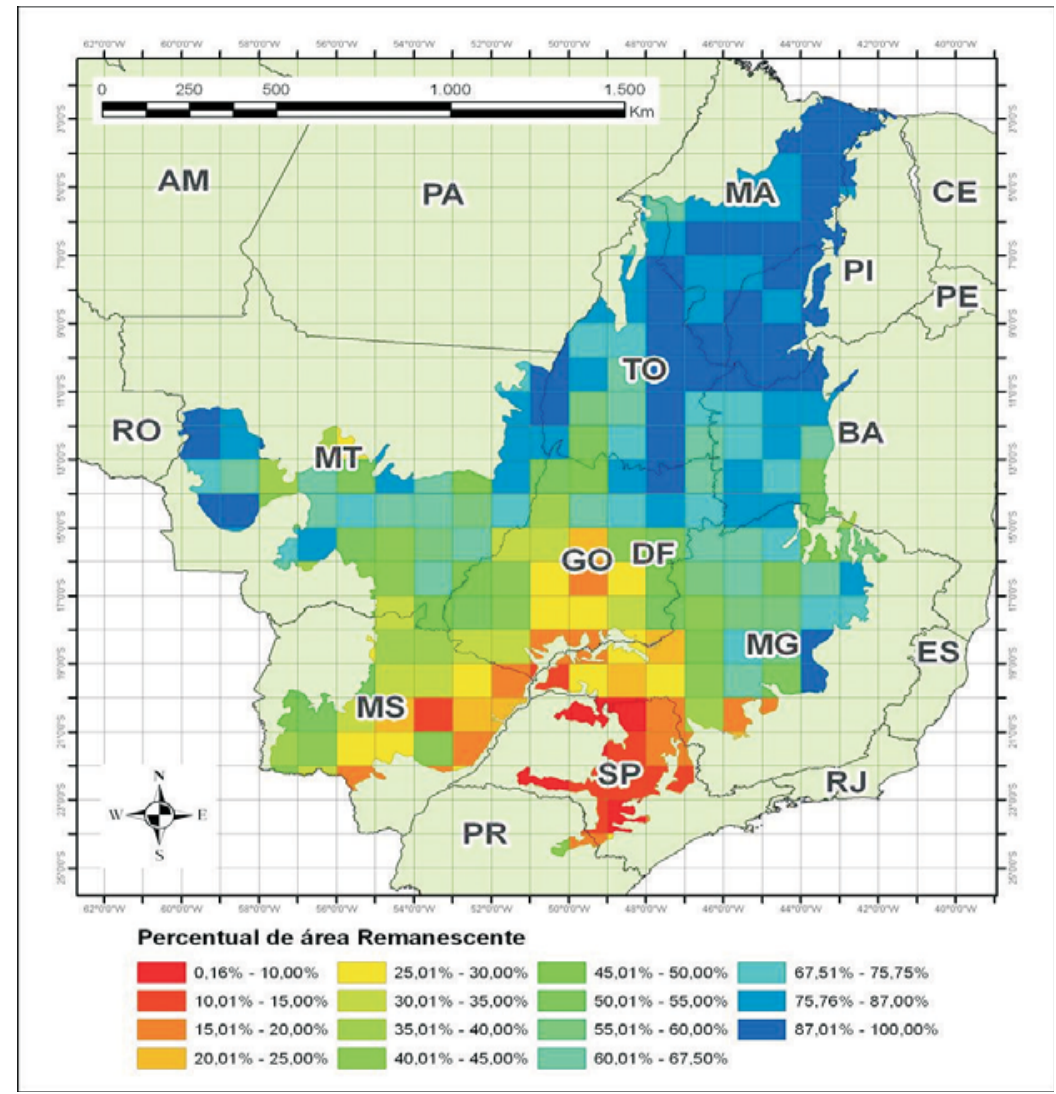

Fonte: Mapa de vegetação remanescente do Cerrado (PROBIO), reamostrado com a escala dotada neste trabalho. Source: Cerrado remnant vegetation map (PROBIO) re-sampled to the scale adopted in this work.

Figura 1 - Mapa do porcentual de área remanescente no bioma Cerrado (células de $1^{\circ} \mathrm{x} 1^{\circ}$ ). Figure 1 - Map of the remnant vegetation percentage for the Cerrado biome $\left(1^{\circ} \times 1^{\circ} \mathrm{cells}\right)$. 
Com vistas a avaliar o efeito das classes de cobertura da terra nos padrões de autocorrelação espacial, as imagens índices de vegetação EVI e NDVI, nos dois períodos considerados, foram mascaradas pixel a pixel e segundo duas categorias (i.e., vegetação remanescente e não-vegetação), com base no mapa de remanescentes do Cerrado. Esse mapa foi produzido a partir de imagens Landsat - TM do ano de $2002^{5}$.

Por fim, e tendo por base a escala comumente adotada nos trabalhos de macroecologia (< 1:2.500.000), os valores de EVI e NDVI, originalmente à Resolução

(a)

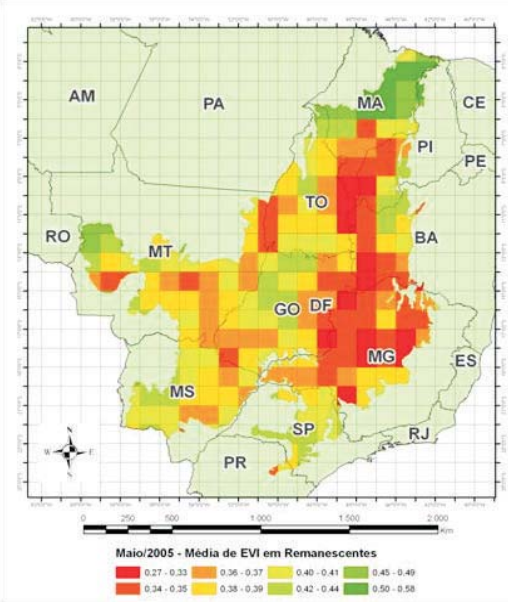

(c)

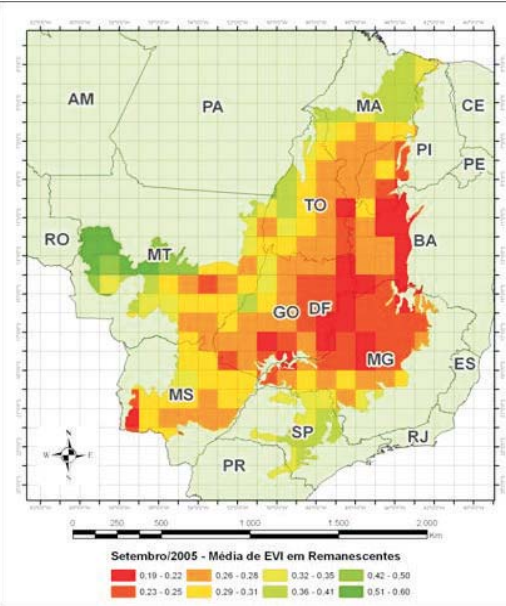

de $250 \mathrm{~m}$, foram agregados para a Resolução de $1^{\circ} \mathrm{x}$ $1^{\circ}$ (Figura 2). Tal redução de escala foi obtida a partir de uma abordagem vetorial, em que o conjunto de pixels contidos em cada uma das células do grid adotado foi representado por um conjunto de atributos pontuais, associados a dada localização $(x, y)$ e a um atributo (z) correspondente aos respectivos valores EVI e NDVI. Assim, um valor médio de EVI e NDVI foi calculado para o conjunto de pontos (pixels) contidos em cada célula de $1^{\circ} \times 1^{\circ}$, sendo o conjunto de pontos médios resultantes espacializado para todo o grid considerado.

(b)

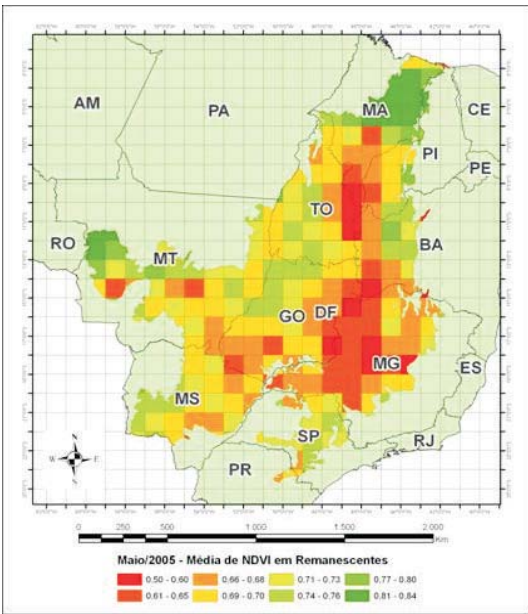

(d)

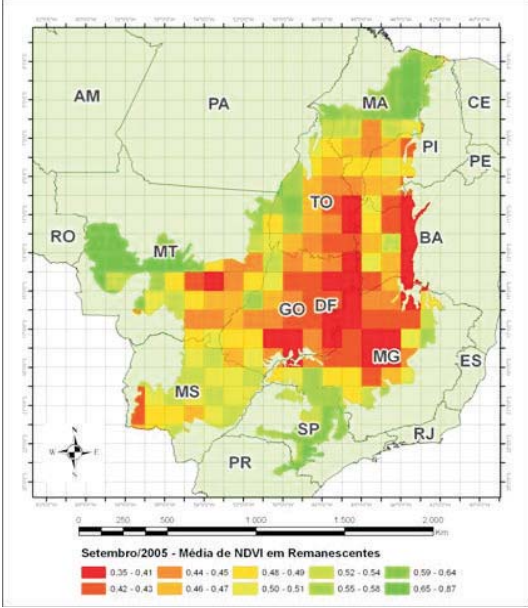

Figura 2 - Valores médios de EVI (a) e NDVI (b) em maio (estação úmida) e de EVI (c) e NDVI (d) em setembro (estação seca), considerando-se apenas a vegetação remanescente do bioma Cerrado.

Figure 2 - Mean values of EVI (a) and NDVI (b) in May (wet season) and of EVI (c) and NDVI (d) in September (dry season), considering only the remnant vegetation of the Cerrado biome.

\footnotetext{
${ }^{5}$ Mapa ainda não publicado, produzido pela Embrapa Cerrados (em colaboração com a Universidade Federal de Uberlândia e Universidade Federal de Goiás), no escopo do projeto PROBIO/MMA/GEF.
} 


\subsection{Medidas de autocorrelação espacial}

A autocorrelação espacial foi descrita pelo coeficiente I de Moran, que é o coeficiente mais usado, medindose a similaridade entre observações de pares de localidades para cada classe de distância. O I de Moran é expresso pela fórmula:

$$
I=\left(\frac{n}{S}\right)\left[\frac{\sum_{i} \sum_{j}\left(y_{i}-\bar{y}\right)\left(y_{j}-\bar{y}\right) w_{i j}}{\sum_{i}\left(y_{i}-\bar{y}\right)^{2}}\right]
$$

em que $n$ é o número de amostras (células de $1^{\circ} \times 1^{\circ}$ ), $y_{i}$ e $y_{j}$ são os valores da variável nas células $i$ e $j, \bar{y}$ é a média de $y$ e $w_{i j}$ é um elemento da matriz $\mathbf{W}$. Nessa matriz, $w_{i j}=1$ se o par $i, j$ das células estiver dentro de dado intervalo de classes de distância (indicando células que estão conectadas nessa classe) e $w_{i j}=$ 0 se não estão nesse intervalo. $S$ é o número de entradas (conexões) da matriz W. Esta matriz, considerando a grade para o Cerrado, tem dimensão igual a 243 linhas por 243 colunas.

Valores positivos de $I$ de Moran indicam autocorrelação positiva, i.e., células espacialmente mais próximas são mais similares; valores negativos de $I$ de Moran indicam autocorrelação negativa, i.e., células espacialmente mais próximas são mais dissimilares do que seria esperado ao acaso (ROSSI e QUÉNÉHERVÉ, 1998; DINIZ-FILHO et al., 2003). Os valores de $I$ de Moran usualmente variam entre - 1,0 e 1,0, indicando máxima autocorrelação negativa e positiva, respectivamente. Porém, esses limites não são necessariamente estáveis, de modo que para comparar os coeficientes $I$ de Moran entre mais de uma série de dados é apropriado para padronizá-los. Isso pode ser feito dividindo-se os valores calculados do $I$ de Moran pelo $I$ de Moran máximo, que pode ser calculado segundo a seguinte fórmula:

$$
\left|I_{\max }(d)\right|=\left(\frac{n}{S_{0}}\right)\left\{\frac{\sum_{i}\left[\sum_{j} w_{i j}\left(y_{j}-\bar{y}\right)\right]^{2}}{\sum_{i}\left(y_{i}-\bar{y}\right)}\right\}^{\frac{1}{2}} .
$$

O valor do $I$ de Moran esperado sob a hipótese nula de ausência de autocorrelação é dado por E(I) $=-1 /(\mathrm{n}-1)$. A significância estatística dos $I$ de Moran foi estabelecida, assumindo-se distribuição normal dos coeficientes, de modo que:

$$
\mathrm{Z}=\mathrm{I}-\mathrm{E}(\mathrm{I}) / \mathrm{e}_{\mathrm{I}}>1,96
$$

em que $e_{\mathrm{I}}$ é o erro do coeficiente, conforme DinizFilho et al. (2003). Valores de $Z>1,96$ indicam um coeficiente significativamente diferente do esperado sob a hipótese nula.

As distâncias espaciais podem ser particionadas em classes discretas, criando sucessivas matrizes $\mathbf{W}$ e permitindo a computação de diferentes valores de I de Moran para a mesma variável. Dessa forma, é possível avaliar o comportamento da autocorrelação como função da distância espacial, em um gráfico chamado de correlograma, que fornece uma descrição do padrão espacial dos dados (DINIZ-FILHO et al., 2003). As análises de autocorrelação espacial foram realizadas utilizandose o software SAM ("Spatial Analysis in Macroecology”), disponível em www.ecoevol.ufg.br/ sam (RANGEL et al., 2006).

A significância geral dos correlogramas foi testada aplicando-se o critério de Bonferroni, de modo que, para um nível de significância $\alpha=0,05$, o correlograma foi considerado significativo (ou seja, apresenta uma estrutura espacial geral) quando pelo menos um dos coeficientes $I$ de Moran foi significativo para um nível de significância $\alpha / k$, sendo $k$ o número de classes de distância usado (neste caso, 12 classes). Um teste $\mathrm{t}$ também foi utilizado para comparar, segundo o critério de Bonferroni, os coeficientes $I$ de Moran (para uma mesma classe de distância), com vistas a estabelecer a significância das diferenças observadas entre os correlogramas.

As análises do padrão espacial dos índices de vegetação foram feitas com os valores médios de NDVI e EVI para maio (estação úmida) e setembro (estação seca) associados ao ponto central de cada célula, considerando-se a cobertura de terra total (vegetação remanescente e não-vegetação) e somente a vegetação remanescente.

\section{RESULTADOS}

Como se pode observar na Figura 1, a qual retrata o mapa do PROBIO (vegetação remanescente e nãovegetação) transformado à escala de análise deste trabalho, as células com maior porcentual de remanescentes estão localizadas nas porções norte e nordeste do bioma Cerrado, desde o norte do Estado de Goiás e oeste da Bahia até o Maranhão e Piauí (limite

R. Árvore, Viçosa-MG, v.32, n.2, p.279-290, 2008 
com a Caatinga), e também nas células localizadas no entorno do Pantanal. As células com menor área de remanescentes estão localizadas no sul do Cerrado, principalmente no Estado de São Paulo.

Em relação aos índices de vegetação das áreas remanescentes, em maio, os valores mais altos do EVI e NDVI foram observados no norte (Maranhão e Piauí), oeste e também no sul (São Paulo). No entanto, os valores mais baixos do NDVI e do EVI foram encontrados no leste do Cerrado, especialmente nos Estados de Goiás, Minas Gerais e Tocantins (Figura 2ab). Em setembro, valores de NDVI e de EVI acima de 0,50 foram observados nas regiões oeste, norte e sul, enquanto na região leste, especialmente nos Estados de Minas Gerais e Bahia, predominam os valores mais baixos (Figura 2cd).

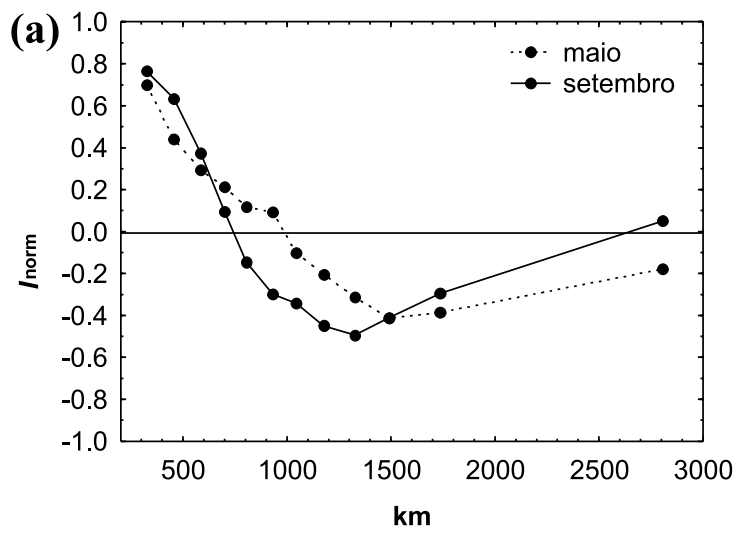

(c)

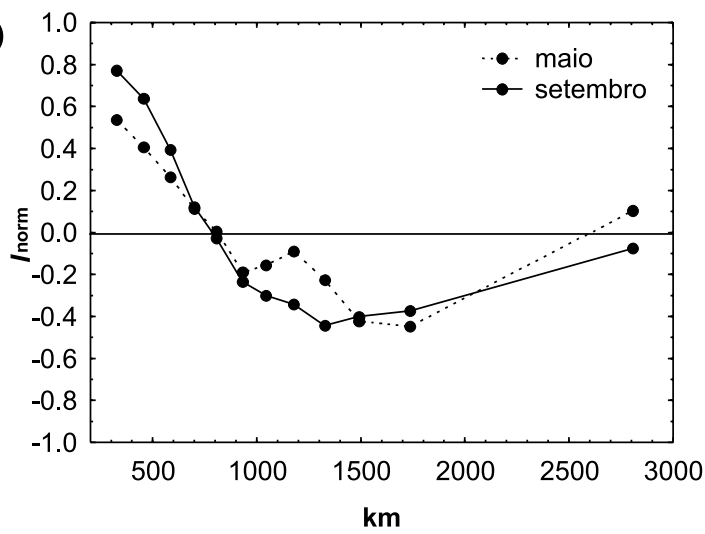

Em relação aos correlogramas, todos foram significativamente diferentes no nível de significância de 0,004 ( $\alpha=0,05 / 12$ classes, aplicando o critério de Bonferroni), mesmo nos casos em que não houve diferenças visuais perceptíveis. A autocorrelação espacial dos dois índices é bastante evidente e, como observado na Figura 3ab, influenciada pela conspícua sazonalidade do bioma Cerrado. No período úmido, tanto o EVI quanto o NDVI, considerando-se a cobertura total, apresentam padrões de autocorrelação negativa a partir de cerca de $900 \mathrm{~km}$, enquanto em setembro a dependência espacial é perceptível até aproximadamente $700 \mathrm{~km}$. Diferenças atribuídas à sazonalidade também são observadas quando somente a cobertura remanescente é considerada. Nesse caso, contudo, os valores de autocorrelação negativa, tanto no período úmido quanto no seco, têm origem nas mesmas classes de distância (Figura $3 \mathrm{~cd}$ ).
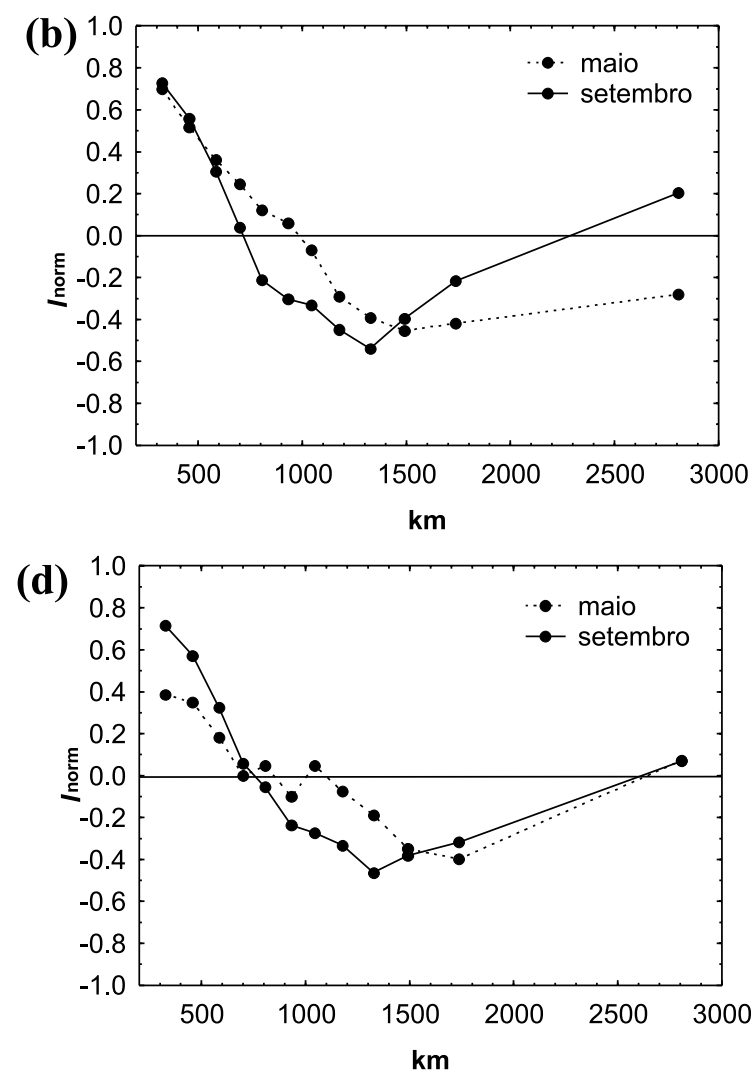

Figura 3 - Correlogramas (classe de distância vs. $I_{\text {norm }}$ ) dos períodos úmido (maio) e seco (setembro) dos valores médios de EVI (a) e NDVI (b), considerando-se a cobertura total (i.e., vegetação remanescente e não vegetação); e dos valores médios de EVI (c) e NDVI (d), considerando-se somente a vegetação remanescente.

Figure 3 - Correlograms (distance class vs. I norm) for the wet (May) and dry (September) periods for the mean values of EVI (a) and NDVI (b), considering total land cover (i.e. remnant vegetation and non-vegetation) and for the mean values of EVI (c) and NDVI (d), considering only the remnant vegetation.

R. Árvore, Viçosa-MG, v.32, n.2, p.279-290, 2008 
Quanto ao índice utilizado, quando se considera a cobertura total, tanto o EVI quanto o NDVI apresentam padrões de autocorrelação espacial semelhantes no período úmido e no período seco (Figura 4ab). Quando se considera apenas a cobertura de vegetação remanescente, esses índices também apresentam padrões espaciais semelhantes em setembro, mas ligeiramente distintos em maio. Nesse caso, o EVI apresenta autocorrelação positiva até distâncias maiores do que o NDVI (Figura 4cd).
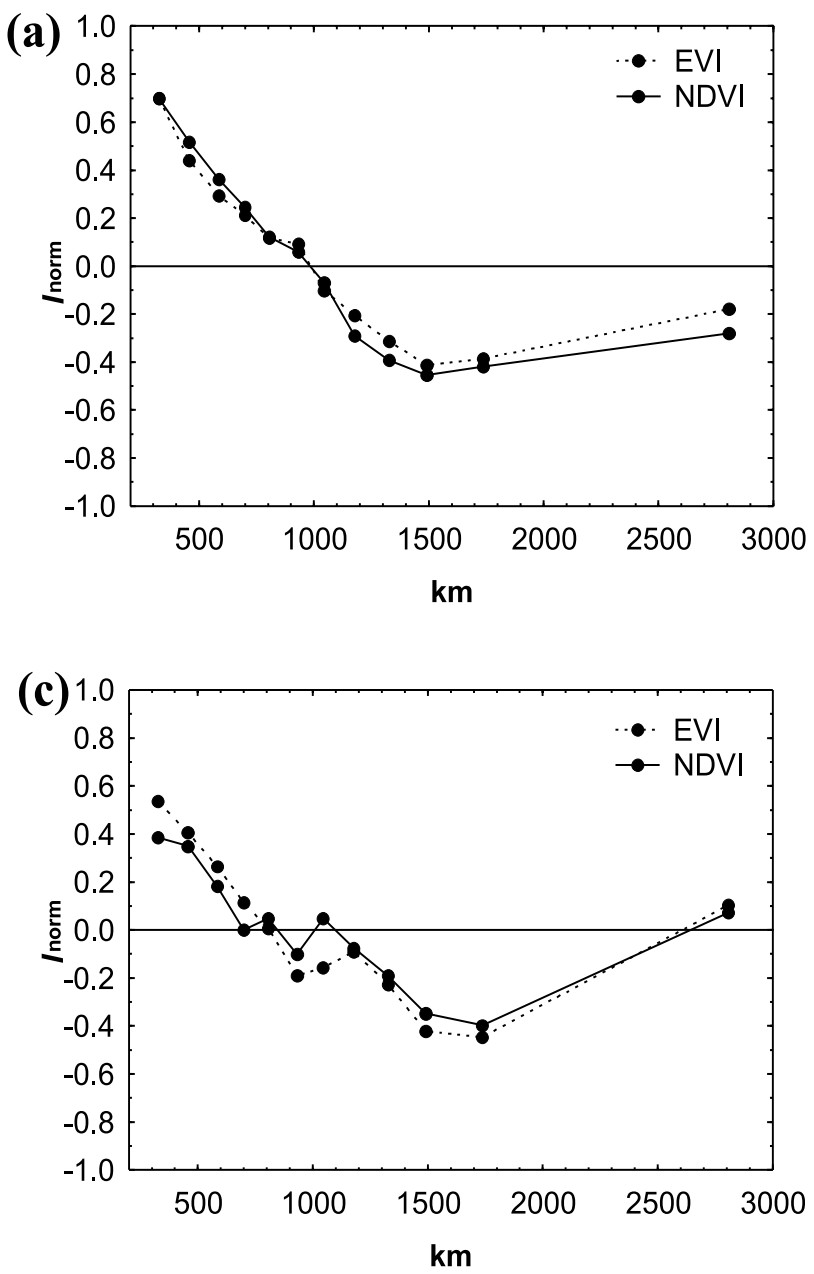

Com relação ao tipo de cobertura (total vs. vegetação remanescente), em maio o EVI apresentou autocorrelação positiva até distâncias maiores quando se considerou a cobertura total. Em setembro, os padrões espaciais foram semelhantes, embora com autocorrelação positiva até distâncias maiores, ao se considerar a cobertura remanescente (Figura 5ab). Já o NDVI mostrou em maio autocorrelação positiva até distâncias maiores para cobertura total, em comparação com a cobertura remanescente. Em setembro, observou-se a situação oposta, i.e., maiores valores de autocorrelação positiva associados à cobertura remanescente (Figura 5cd).
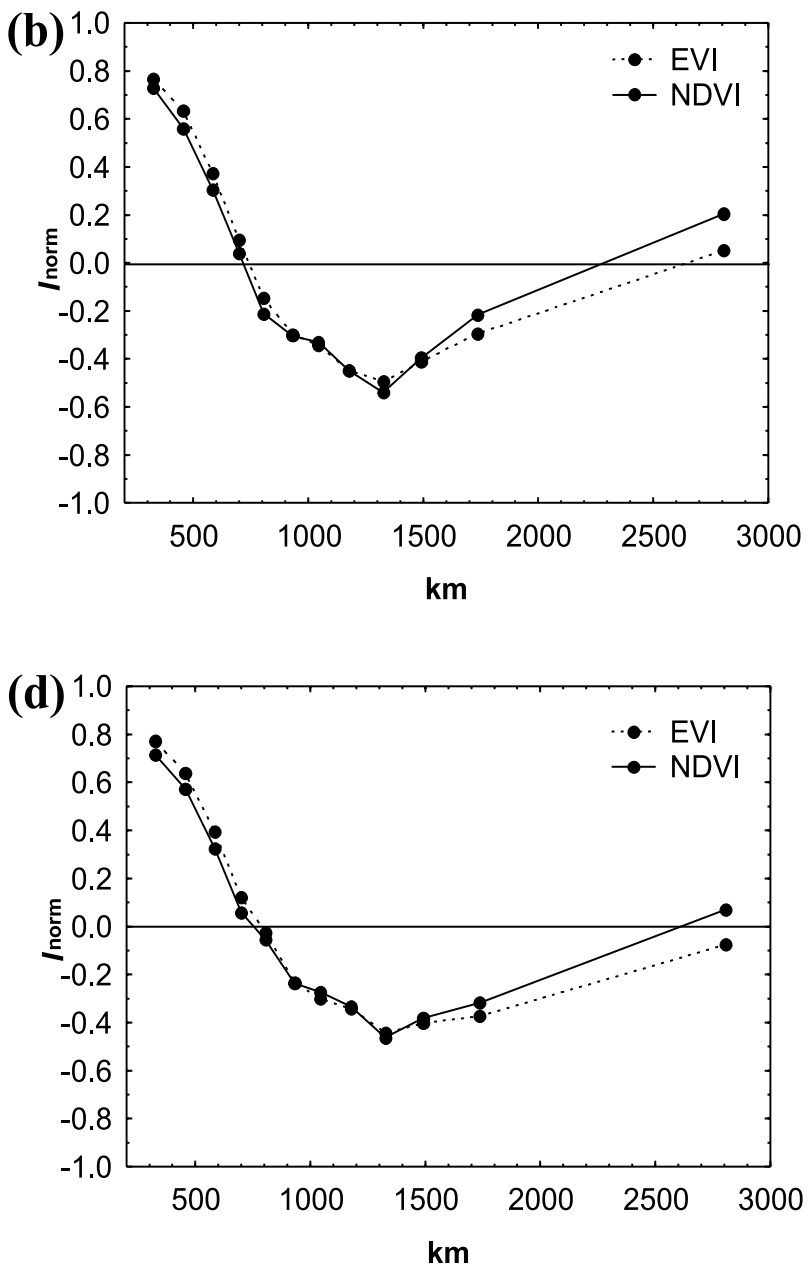

Figura 4 - Correlogramas (classe de distância vs. $I_{\text {norm }}$ ) dos NDVI e EVI em maio (estação úmida) (a) e em setembro (estação seca) (b), considerando-se a cobertura total (i.e., vegetação remanescente e não vegetação); e dos NDVI e EVI em maio (estação úmida) (c) e em setembro (estação seca) (d), considerando-se somente a vegetação remanescente.

Figure 4-Correlograms (distance class vs. I ${ }_{\text {norm }}$ ) for NDVI and EVI in May (wet season)(a) and in September (dry season) (b), considering the total land cover (i.e. remnant vegetation and non-vegetation) for NDVI and EVI in May (wet season)(c) and in September (dry season) (d), considering only the remnant vegetation. 

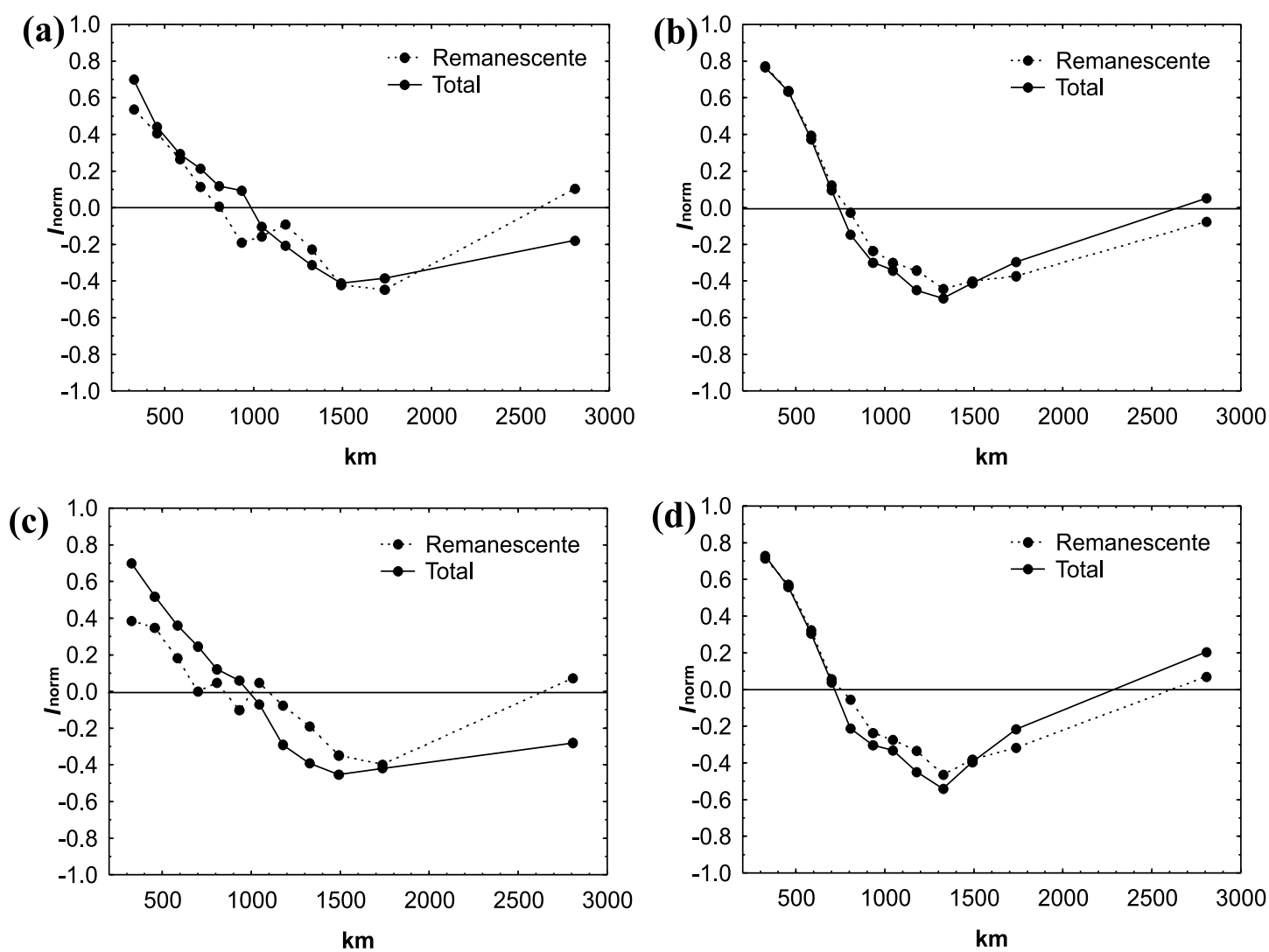

Figura 5 - Correlogramas (classe de distância $v s . I_{\text {norm }}$ ) dos valores médios de EVI em maio (a) e setembro (b), considerandose a cobertura total (i.e., vegetação remanescente e não vegetação) e somente a vegetação remanescente, e dos valores médios de NDVI em maio (c) e setembro (d), considerando-se a cobertura total (i.e., vegetação remanescente e não vegetação) e somente a vegetação remanescente.

Figure 5 - Correlograms (distance class vs. $I_{\text {norm }}$ ) for the mean values of EVI in May (a) and September (b), considering total cover (i.e. remnant vegetation and non-vegetation) and only the remnant vegetation and for the mean values of NDVI in May (c) and September (d), considering total land cover (i.e. remnant vegetation and nonvegetation) and only the remnant vegetation.

\section{DISCUSSÃO}

Os padrões observados no comportamento dos índices de vegetação EVI e NDVI ao longo do Cerrado, considerando-se tanto o uso total da terra quanto somente a vegetação remanescente, podem ser esperados devido à escala analisada neste estudo. Cada célula do grid em que foi dividido o Cerrado tem número muito grande de pixels (aproximadamente 200.000); desse modo, os valores de EVI e NDVI em cada célula estão muito "diluídos", tendendo a uma homogeneização dos valores médios e gerando, portanto, o comportamento observado. Mesmo assim, eles ainda são capazes de indicar variações importantes, correlacionadas com a biomassa vegetal, como os maiores valores em áreas do norte do Maranhão e em áreas extremas do Mato Grosso e baixos valores na área central de Goiás e Minas Gerais.

Os valores mais baixos observados na estação seca e mais altos na estação úmida são esperados e concordam com o que já foi descrito por outros autores para o Cerrado (FERREIRA et al., 2003). Esse padrão ocorre porque os índices são proporcionais à atividade fotossintética, apresentando valores maiores quando a atividade fotossintética é maior e há mais biomassa verde.

As formas dos correlogramas (de maneira geral, com autocorrelação positiva nas distâncias mais curtas e também nas distâncias maiores) indicam que o EVI e o NDVI apresentam estrutura espacial em manchas 
(LEGENDREe FORTIN, 1989). De fato, podem-se observar, nos mapas da Figura 2 abcd, valores mais altos nas bordas e mais baixos no centro.

Variações no comportamento espacial do EVI e do NDVI em função da sazonalidade e do tipo de cobertura vegetal (vegetação remanescente vs. não-vegetação) foram bem evidentes. Considerando-se apenas a cobertura remanescente, os padrões de autocorrelação dos dois índices, tanto em setembro quanto em maio, mudam aproximadamente na mesma distância. Entretanto, quando a cobertura total é considerada (i.e., uso e vegetação remanescente), os padrões de autocorrelação do EVI e NDVI na estação seca tendem a mudar a distâncias mais curtas do que na estação úmida. Essas diferenças estão diretamente relacionadas à maior diversidade de paisagens presentes na "cobertura total", realçadas na estação seca, bem como ao fato de que na estação úmida a cobertura "verde", predominante, se distribui mais uniformemente.

Quando se analisa, no entanto, o efeito do tipo de cobertura (vegetação remanescente $v s$. nãovegetação), padrões de autocorrelação espacial distintos, tanto para EVI quanto para NDVI, são observados preferencialmente em maio. Essas diferenças, menos acentuadas do que aquelas associadas ao contraste sazonal, são caracterizadas pela menor dependência espacial dos índices de vegetação relacionada à cobertura remanescente, cujos padrões de autocorrelação espacial mudam a distâncias aproximadas de $800 \mathrm{~km}$, enquanto os valores dos índices associados à cobertura total são espacialmente correlacionados até distâncias de aproximadamente $900 \mathrm{~km}$.

Diniz-Filho et al. (2003) mostraram uma padrão de autocorrelação espacial de evapotranspiração e de relevo compatível com manchas de até $1.900 \mathrm{~km}$ e que concordam com as manchas na distribuição da riqueza de espécies de aves. Neste estudo, o conjunto das informações resultantes dos correlogramas indica uma estrutura em manchas com tamanho entre 800 e 900 $\mathrm{km}$, na maioria das situações analisadas. A interpretação mais simples que se pode ter desse resultado é a existência de condições que determinam um padrão de valores para os índices de vegetação com efeito que se estende espacialmente por entre 800 e $900 \mathrm{~km}$. O conhecimento atual da variação global nas taxas de fotossíntese (GENTRY e EMMONS, 1987; KERKHOFF et al., 2005) evidencia que a variação nos índices de vegetação pode estar associada à intensidade luminosa, temperatura, distribuição de chuvas, distribuição de tipos de solos e relevo. Dessas variáveis, possivelmente apenas solos e o relevo podem apresentar modelo de distribuição em manchas compatível com os observados neste estudo. Entretanto, não se pode deixar de observar que os valores dos índices de vegetação são afetados também pelo estado de conservação das áreas, e também a distribuição das manchas de maior intensidade de alteração ambiental afeta os padrões espaciais aqui verificados.

Outra visão sobre esses resultados registra um viés mais metodológico. Um valor do índice $I$ de Moran é igual a zero (ou seja, há ausência de autocorrelação espacial), indicando distâncias em que os dados são independentes. A independência entre amostras é essencial nos estudos que buscam explicações para os padrões espaciais, como os encontrados neste estudo e que se baseiam no uso de regressão por quadrados mínimos ordinários (DINIZ-FILHO et al., 2003; HAWKINS et al., 2007). Com base nesta pesquisa, considerouse que a independência só é atingida utilizando-se células com distâncias entre 800 e $900 \mathrm{~km}$. A alternativa a esse problema é o uso da regressão com autocorrelação espacial (Spatial Autocorrelation Regression, SAR), que já é disponível em vários pacotes estatísticos (RANGEL et al., 2006).

É importante ressaltar que os padrões de autocorrelação espacial descritos neste estudo são dependentes da escala de análise. Considerando o grid utilizado (i.e., $1^{\circ} \times 1^{\circ}$ ) e a resolução espacial original das imagens MODIS (250 m), há uma significativa uniformização dos valores dos índices de vegetação. Assim, e considerando escalas/resoluções maiores, espera-se que outros padrões de autocorrelação, de menor dependência e similaridade espacial, sejam observados.

\section{CONCLUSÃO}

Este trabalho avaliou, na escala comumente adotada em estudos de macroecologia, os padrões de autocorrelação espacial dos índices de vegetação EVI e NDVI. Esses índices evidenciaram comportamentos semelhantes ao longo do bioma Cerrado e entre as estações seca e úmida. Os valores mais altos e os mais baixos dos dois índices foram observados nas mesmas regiões.

R. Árvore, Viçosa-MG, v.32, n.2, p.279-290, 2008 
Os valores apresentados por esses índices em escalas inferiores a 1:2.500.000 são correlacionados até uma distância aproximada de $800 \mathrm{~km}$, o que pode ser resultado da distribuição de fatores ambientais como solo e relevo, além de possíveis perturbações causadas pela alteração da cobertura vegetal. Esses padrões de similaridade espacial são principalmente influenciados pelo contraste sazonal apresentado pelas fitofisionomias do bioma Cerrado. Em menor intensidade, a dependência espacial apresentada por esses índices também tende a variar em função dos padrões de cobertura da terra e do tipo de índice considerado (i.e., EVI ou NDVI).

\section{AGRADECIMENTOS}

Ao Projeto Integração de Informações Espaciais para o Planejamento e Gestão Estratégica do Bioma Cerrado (CNPq/Edital Universal 481939/2004-0) e ao Programa PRONEX para estabelecimento de áreas prioritárias para conservação no Cerrado (CNPq/ SECTEC-GO, Proc. 23234156); também a P. De Marco e três revisores anônimos, por suas sugestões a uma versão preliminar deste artigo.

\section{REFERÊNCIAS}

CALEGARIO, N. et al. Eucalyptus stand growth estimate based on multilevel linear mixed-effects models. Revista Árvore, v.29, n.2, p.251-264, 2005.

DINIZ-FILHO, J. A. F.; SANT’ANA, C. E. R. Macroecological analysis of South American owls (Strigiformes) using phylogenetic independent contrasts. Journal of Comparative Biology, v.3, n.1, p.27-32, 1998.

DINIZ-FILHO, J. A. F.; BINI, L. M.; HAWKINS, B. A. Spatial autocorrelation and red herrings in geographical ecology. Global Ecology and Biogeography, v. 12, n.1, p.53-64, 2003.

DINIZ-FILHO, J. A. F. et al. Conservation biogeography of anurans in Brazilian Cerrado. Biodiversity and Conservation, v.16, n.4, p.997-1008, 2007.

FERREIRA, L. G. et al. Seasonal landscape and spectral vegetation index dynamics in the Brazilian Cerrado: An analysis within the LargeScale Biosphere-Atmosphere Experiment in Amazônia (LBA). Remote Sensing of Environment, v.87, n.4, p.534-550, 2003.

R. Árvore, Viçosa-MG, v.32, n.2, p.279-290, 2008
FERREIRA, L. G.; HUETE, A. R. Assessing the seasonal dynamics of the Brazilian Cerrado vegetation through the use of spatial vegetation indices. International Journal of Remote Sensing, v.25, n.10, p.1837-1860, 2004.

FERREIRA, L. G. et al. Optical characterization of the Brazilian Savanna physiognomies for improved land cover monitoring of the cerrado biome: Preliminary assessments from an airborne campaign over an LBA core site. Journal of Arid Environments, v.56, n.3, p.425-447, 2004.

FERREIRA, N. C. et al. An operational deforestation mapping system using MODIS data and spatial context analysis. International Journal of Remote Sensing, 2006a (in press).

FERREIRA, M. E. et al. Análise da sazonalidade de paisagens antrópicas e nativas do bioma Cerrado através dos produtos MODIS índices de vegetação, área foliar e atividade fotossintética. Revista Brasileira de Geofísica. 2006b (in press).

GAO, X. et al. Optical-biophysical relationships of vegetation spectra without background contamination. Remote Sensing of Environment, v.74, n.3, p.609-620, 2000.

GENTRY, A. H.; EMMONS, L. H. Geographical variation in fertility, phenology, and composition of the understory of Neotropical forests. Biotropica, v.19, n.3, p.216-227, 1987.

GOMES, M. P.; SANO, E. E.; FERREIRA, L. G. Desmatamento no Estado de Goiás: possibilidades de monitoramento com sensores orbitais de resolução espacial moderada. Revista Brasileira de Geofísica. 2006 (in press).

GOWARD, S. N. et al. Normalized difference vegetation index measurements from the Advanced Very High Resolution Radiometer. Remote Sensing of Environment, v.35, n.2-3, p.257-277, 1991.

HAWKINS, B. A.; DINIZ-FILHO, J. A. F.; SOELLER, S. A. Water links the historical and contemporary components of Australian bird diversity gradient. Journal of Biogeography, v.32, n.6, p.1035-1042, 2005. 
HAWKINS, B. A. et al. Red herrings revisited: spatial autocorrelation and parameter estimation in geographical ecology. Ecography, v.30, n.3, p.375-384, 2007.

HUETE, A. R. et al. Overview of the radiometric and biophysical performance of the MODIS vegetation indices. Remote Sensing of Environment, v. 83, n.1-2, p.195-213, 2002.

HUETE, A. R. et al. A comparison of vegetation indices over a global set of TM images for EOSMODIS. Remote Sensing of

Environment, v.59, n.3, p.440-451, 1997.

HURLBERT, A. H.; HASKELL, J. P. The effect of energy and seasonality on avian species richness and community composition. The American Naturalist, v.161, n.1, p.83-97, 2003.

INSTITUTOBRASILEIRODEGEOGRAFIAE ESTATÍSTICA - IBGE. Mapa de biomas do Brasil. 2004.

JACKSON, R. D.; HUETE, A. R. Interpreting vegetation indices. Preventive Veterinary Medicine, v.11, n.3-4, p.185-200, 1991.

JUSTICE, C. O. et al. The Moderate Resolution Imaging Spectroradiometer (MODIS): Land remote sensing for global change research. IEEE Transactions on Geoscience and Remote Sensing, v.36, p.1-22, 1998.

KERKHOFF, A. J. et al. Plant allometry, stoichiometry and the temperature-dependence of primary productivity. Global Ecology and Biogeography, v.14, n.6, p.585-598, 2005.

KERR, J. T.; OSTROVSKY, M. From space to species: ecological applications for remote sensing. Trends in Ecology and Evolution, v.18, n.6, p.299-305, 2003.

KOY, K. et al. Percentage canopy cover - using Landsat imagery to delineate habitat for Myanmar's endangered Eld's deer (Cervus eldi). Animal Conservation, v.8, n.3, p.289-296, 2005.

LACERDA, C. M. B.; KAGEYAMA, P. Y. Spatial genotype structure of two natural populations of Myracrodruon urundeuva M. Allemão in a semiarid region of Brazil. Revista

Árvore, v.27, n.2, p.145-150, 2003.
LEGENDRE, P.; FORTIN, M.-J. Spatial pattern and ecological analysis. Vegetatio, v.80, n.2, p.107-138, 1989.

LEGENDRE, P. Spatial autocorrelation: trouble or new paradigm? Ecology, v.74, n.6, p.1659-1673, 1993.

LENNON, J. J. Red-shifts and red herrings in geographical ecology. Ecography, v.23, n.1, p.101-113, 2000.

LIU, H. Q.; HUETE, A. A feedback based modification of the NDVI to minimize canopy background and atmospheric noise. IEEE Transactions on Geoscience and Remote Sensing, v.33, n.2, p.457-465, 1995.

MOODY, A.; STRAHLER, A. H. Characteristics of composited AVHRR data and problems in their classification. International Journal of Remote Sensing, v.15, n.17, p.3473-3491, 1994.

MIURA, T. et al. Vegetation detection through smoke-filled AVIRIS images: An assessment using MODIS bandpasses. Journal of Geophysical Research, v. 103, n.D24, p.32.001-32.012, 1998.

MIURA, T. et al. An error and sensitivity analysis of atmospheric resistant vegetation indices derived from dark target-based atmospheric correction, Remote Sensing of

Environment, v.78, n.3, p.284-298, 2001.

RATANA, P.; HUETE, A.; FERREIRA, L. G. Analysis of Cerrado physiognomies and conversion in the MODIS seasonal-temporal domain. Earth Interactions, v.9, n.3, p.1-22, 2005.

RANGEL, T. F. L. V. B.; DINIZ-FILHO, J. A. F.; BINI, L. M. Towards an integrated computational tool for spatial analysis in macroecology and biogeography. Global Ecology and Biogeography, v.15, n.4, p.321-327, 2006.

RODRIGUEZ, M. A.; LOPEZ-SAÑUDO, I. L.; HAWKINS, B. A. The geographic distribution of mammal body size in Europe. Global Ecology and Biogeography, v. 15, n.2, p.173-181, 2006.

R. Árvore, Viçosa-MG, v.32, n.2, p.279-290, 2008 
ROSSI, J.-P.; QUÉNÉHERVÉ, P. Relating species density to environmental variables in presence of spatial autocorrelation: a study case on soil nematodes distribution. Ecography, v.21, n.2, p.117-123, 1998.

RUGGIERO, A.; KITZBERGER, T. Environmental correlates of mammal species richness in South America: Effects of spatial structure, taxonomy and geographic range. Ecography, v.27, n.4, p.401-416, 2004.

SELMI, S.; BOULINIER, T. Ecological biogeography of Southern Ocean Islands: The importance of considering spatial issues. The American Naturalist, v.158, n.4, p.426-437, 2001.
TOGNELLI, M. F.; KELT, D. A. Analysis of determinants of mammalian species richness in South America using spatial autoregressive models. Ecography, v.27, n.4, p.427-436, 2004.

WANG, Z. X.; LIU, C.; HUETE, A. From AVHRRNDVI to MODIS-EVI: Advances in vegetation index research. Acta Ecologica Sinica, v.23, n.5, p.979-988, 2003.

WESSELS, K. J. et al. Mapping regional land cover with MODIS data for biological conservation: Examples from the Greater Yellowstone Ecosystem, USA and Pará State, Brazil. Remote Sensing of Environment, v.92, n.1, p.67-83, 2004. 